ORIGINAL ARTICLE

\title{
Expression of SRC-1, AIB1, and PEA3 in HER2 mediated endocrine resistant breast cancer; a predictive role for SRC-1
}

\section{F J Fleming, E Myers, G Kelly, T B Crotty, E W McDermott, N J O'Higgins, A D K Hill, L S Young}

J Clin Pathol 2004;57:1069-1074. doi: 10.1136/icp.2004.016733

See end of article for authors' affiliations

....................

Correspondence to: Dr L S Young, Department of Surgery, Conway Institute of Biomolecular and Biomedical Research, University College Dublin, Dublin 4, Ireland; leonie. young@ucd.ie

Accepted for publication 11 May 2004
Background: In human breast cancer, the growth factor receptor HER2 is associated with disease progression and resistance to endocrine treatment. Growth factor induced mitogen activated protein kinase activity can phosphorylate not only the oestrogen receptor, but also its coactivator proteins AIB 1 and SRC-1.

Aim: To determine whether insensitivity to endocrine treatment in HER2 positive patients is associated with enhanced expression of coactivator proteins, expression of the HER2 transcriptional regulator, PEA3, and coregulatory proteins, AIB1 and SRC-1, was assessed in a cohort of patients with breast cancer of known HER2 status.

Methods: PEA3, AIB1, and SRC-1 protein expression in 70 primary breast tumours of known HER2 status (HER2 positive, $n=35$ ) and six reduction mammoplasties was assessed using immunohistochemistry. Colocalisation of PEA3 with AIB1 and SRC-1 was determined using immunofluorescence. Expression of PEA3, AIB1, and SRC-1 was correlated with clinicopathological parameters.

Results: In primary breast tumours expression of PEA3, AIB1, and SRC-1 was associated with HER2 status $(p=0.0486, p=0.0444$, and $p=0.0012$, respectively). In the HER2 positive population, PEA3 expression was associated with SRC-1 $(p=0.0354)$, and both PEA3 and SRC-1 were significantly associated with recurrence on univariate analysis $(p=0.0345 ; p<0.0001)$. On multivariate analysis, SRC-1 was significantly associated with disease recurrence in HER2 positive patients $(p=0.0066)$.

Conclusion: Patients with high expression of HER2 in combination with SRC-1 have a greater probability of recurrence on endocrine treatment compared with those who are HER2 positive but SRC-1 negative. SRC-1 may be an important predictive indicator and therapeutic target in breast cancer.
$\mathrm{T}$ he HER2 gene encodes a receptor tyrosine kinase that is structurally related to the epidermal growth factor receptor (EGFR) family, and which acts through downstream signalling cascades, including the Ras and phosphoinositide 3' kinase pathways, to alter gene expression. In human breast cancer, overexpression of HER2 (c-erbB2) is associated with a poor prognosis. ${ }^{1}$ Expression of this oncogene has been found to correlate with enhanced tumorigenicity, increased metastatic potential, and resistance to chemotherapy. ${ }^{2}$ The Ets transcription factor, PEA3, is thought to play an essential role in HER2 mediated oncogenesis. Although a large body of evidence exists to suggest that PEA3 is central to the transcriptional regulation of HER2, the direction of this modulation remains controversial. ${ }^{45}$ Ex vivo observations that PEA3 is overexpressed in $93 \%$ of HER2 positive breast tumours, and molecular studies showing that expression of dominant negative PEA3 in the mammary gland of mouse mammary tumour virusneu transgenic mice delays tumour onset, ${ }^{67}$ suggest a positive role for PEA3 in the transcriptional regulation of HER2.

"Although a large body of evidence exists to suggest that
PEA3 is central to the transcriptional regulation of HER2,
the direction of this modulation remains controversial"

Both molecular and clinical studies have associated raised HER2 values in oestrogen receptor (ER) positive breast tumours with a decreased response to endocrine treatment, such as tamoxifen. ${ }^{8}$ It has been proposed that increased signalling from the EGFR/HER2 family, activating the mitogen activated protein (MAP) kinase pathway, results in phosphorylation, not only of ER, but also of essential coactivator proteins, including AIBl (amplified in breast cancer 1$).{ }^{10}$ The coactivator protein AIBl, along with SRC-1 (steroid receptor coactivator 1 ), are both members of the pl60 family of coactivator proteins, whose expression is increased in breast cancer. ${ }^{11}{ }^{12}$ Studies by Osborne et al indicate that high expression of AIBl can reduce the antagonist activity of tamoxifen bound ER in patients with breast cancer, leading ultimately to ineffective endocrine treatment. ${ }^{10}$ Furthermore, we have recently described a positive correlation between SRC-1 and tumour recurrence in patients with breast cancer who are on endocrine treatment. ${ }^{12}$

We hypothesise that HER2, through its transcriptional regulator PEA3, contributes to endocrine resistance by potentiating steroid coactivator proteins. Therefore, we predict that in HER2 positive patients, insensitivity to endocrine treatment associates with enhanced expression of coactivator proteins. To test this, we determined the expression of the HER2 transcriptional regulator, PEA3, and the coregulatory proteins, AIBl and SRC-1, in a cohort of patients with breast cancer of known HER2 status.

Abbreviations: AIB1, amplified in breast cancer 1; EGFR, epidermal growth factor receptor; ER, oestrogen receptor; MAP, mitogen activated protein; PBS, phosphate buffered saline; SRC-1, steroid receptor coactivator-1 
Table 1 Clinicopathological parameters of the HER2 positive and HER2 negative groups

\begin{tabular}{lllll}
\hline & Total group (70) & HER2 positive (\%) & HER2 negative (\%) & p Value \\
\hline Mean (SE) age & $47.6(1.06)$ & $47.6(1.51)$ & $47.6(1.51)$ & 1.000 \\
Mean (SE) tumour size (mm) & $39.77(1.83)$ & $39.8(2.61)$ & $39.74(2.61)$ & 0.9877 \\
Histological grade & 35 & $12(34 \%)$ & $23(66 \%)$ & 0.0162 \\
$\quad$ Grades I and II & 35 & $23(66 \%)$ & $12(34 \%)$ & 1.0000 \\
$\quad$ Grade III & 45 & $23(66 \%)$ & $22(63 \%)$ & \\
Axillary lymph node positivity & &
\end{tabular}

Continuous variables were analysed using the two sample $t$ test. Nominal variables were analysed using Fisher's exact test.

\section{MATERIALS AND METHODS Patient selection}

HER2 positive tumours from patients who received tamoxifen treatment were selected for our study. Each case was matched with a HER2 negative patient of similar age and tumour size at time of diagnosis. HER2 status was evaluated using the Dako (Glostrup, Denmark) HercepTest immunocytochemical assay. Scoring was assessed according to the manufacturer's instructions. A score was assigned according to the intensity and pattern of cell membrane staining: 0 to +1 , no staining, or staining in $<10 \%$ of cells; +2 , weak to moderate staining in $>10 \%$ of cells; +3 , strong staining in $>10 \%$ of cells. In tumour samples scoring +2 with the Hercept test, HER2 status was confirmed by fluorescent in situ hybridisation using the Vysis kit (Abbot Laboratories, Maidenhead, Berkshire, UK) according to the manufacturer's instructions. Patients who received neoadjuvant chemotherapy or endocrine treatment were not included. Histologically normal breast tissue specimens were obtained from six patients who underwent reduction mammoplasties. All patients received chemotherapy and tamoxifen $(20 \mathrm{mg} /$ day $)$ for a maximum of five years. This was discontinued only in those patients who suffered a relapse while on endocrine treatment. In those patients who were ER negative, tamoxifen was prescribed on the basis of the fact that the patients were progesterone receptor positive. ER $\alpha$ immunohistochemistry, as described below, confirmed the postoperative ER status in all cases.

\section{Clinicopathological parameters}

Variables analysed included tumour size, tumour grade, presence of lymphovascular invasion, and axillary node status. A recurrence was defined as any local (chest wall) or systemic (visceral or bone metastasis) recurrence during the follow up period.

\section{Immunohistochemistry}

Tissue sections ( $5 \mu \mathrm{m}$ thick) were cut from paraffin wax embedded breast tumour tissue and reduction mammoplasty blocks and mounted on Superfrost Plus slides (BDH, Poole, Dorset, UK). Sections were dewaxed, rehydrated, and washed in phosphate buffered saline (PBS). Endogenous peroxidase was blocked using 3\% hydrogen peroxidase in PBS for 10 minutes. Antigen retrieval was performed by immersing sections in $0.6 \mathrm{M}$ citrate buffer and microwaving on high power for seven minutes. Antigens were detected using the Vectastain Elite kit (Vector Laboratories, Burlingame, California, USA), according to the manufacturer's instructions. Briefly, sections were blocked in serum for 90 minutes. Sections were incubated with the following primary antibodies: rabbit antihuman $\mathrm{ER} \alpha(1 \mu \mathrm{g} / \mathrm{ml}$; Novacastra, Newcastle upon Tyne, UK), goat antihuman SRC-1 ( $1 \mu \mathrm{g} /$ $\mathrm{ml})$, mouse antihuman PEA3 $(10 \mu \mathrm{g} / \mathrm{ml})$, and rabbit antihuman AIBl ( $1 \mu \mathrm{g} / \mathrm{ml}$; Santa Cruz Technology, Santa Cruz, California, USA) for 60 minutes at room temperature. Sections were subsequently incubated with the corresponding biotin labelled secondary antibody (1/2000 dilution) for
30 minutes, followed by peroxidase labelled avidin-biotin complex. Sections were developed in 3,3' - diaminobenzidine tetrahydrochloride and counterstained with haematoxylin. Negative controls were performed using matched IgG controls (Dako). Sections were examined under a light microscope. Immunostained slides were scored for ER $\alpha$, SRC-1, PEA3, and AIBl using the Allred scoring system. ${ }^{13}$ Each entire slide was evaluated using light microscopy. A proportion score was assigned, which represented the estimated proportion of positively stained tumour cells (none, $0 ;<1 \%, 1 ;>1 \%$ to $<10 \%, 2 ;>10 \%$ to $<33 \%, 3$; $>33 \%$ to $<66 \%, 4 ;>66 \%, 5)$. An intensity score was assigned that represented the average intensity of the positive tumour cells (none, 0; weak, 1; intermediate, 2; strong, 3). The proportion and intensity scores were then added to obtain a total score, which ranged from 0 to 8 . A total score greater than 2 was taken to indicate positivity. Two independent observers, blinded to HER2 status and without knowledge of prognostic factors, scored the slides. The interobserver correlation coefficient for the reliability of each variable was computed, together with a $95 \%$ lower confidence bound. These coefficients and the confidence bounds ranged from 0.824 to 0.914 and from 0.743 to 0.871 , respectively. This level of agreement indicated a high level of interobserver reliability. ${ }^{14}$

\section{Immunofluorescent microscopy}

Breast cancer sections were prepared as above and incubated in sheep serum for 60 minutes. Mouse antihuman PEA3 ( $100 \mu \mathrm{g} / \mathrm{ml}$ dilution with PBS in $10 \%$ human serum) was placed on each slide for 90 minutes. The sections were rinsed in PBS and incubated with the corresponding secondary fluorochrome conjugated antibody $(100 \mu \mathrm{g} / \mathrm{ml}$; SigmaAldrich, Steinheim, Germany) for 60 minutes. The slides were rinsed in PBS and blocked in rabbit serum (SRC-1) or goat serum (AIBI) for 90 minutes, then washed with PBS. Each slide was incubated with either goat antihuman SRC-1 or rabbit antihuman AIBl (both $10 \mu \mathrm{g} / \mathrm{ml}$ with PBS in $10 \%$ human serum) for 90 minutes. The slides were incubated with the corresponding fluorochrome conjugated antibody $(100 \mu \mathrm{g} / \mathrm{ml})$ for 60 minutes. Sections were rinsed in PBS and mounted using fluorescent mounting media (Dako). Sections were examined under a fluorescent microscope. Negative controls were performed using matched IgG.

\section{Statistical analysis}

Continuous variables were assessed using a two sample $t$ test. Fisher's exact test was used in the comparison of two proportions throughout or equivalently to test for association in $2 \times 2$ tables. Where comparisons were made on variables that were correlated, the $\mathrm{p}$ value for each comparison was adjusted using Bonferroni's inequality. A Cox proportional hazards model was used to find significant predictors of disease free survival time. The predictors included in the model were: HER2, axillary status, size of tumour, grade of tumour, PEA3, AIBI, ER $\alpha, \mathrm{ER} \beta$, and SRC-1. A stepwise procedure was used to find the best model. Kaplan-Meier 
A

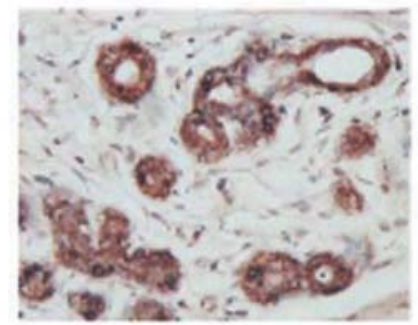

PEA3

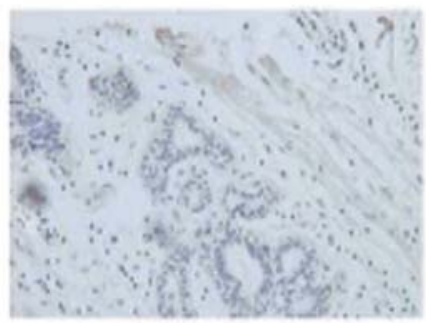

$\lg G$ control

B

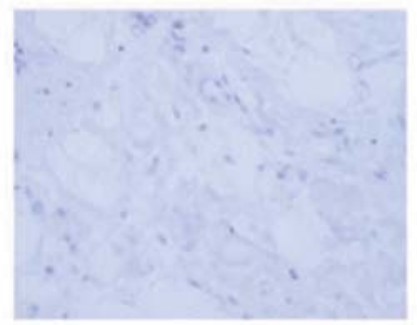

PEA3

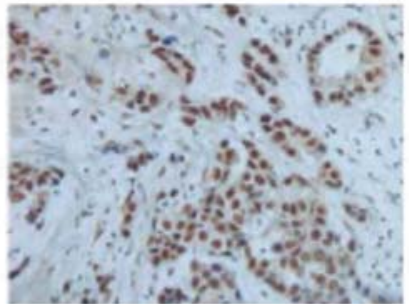

AlB-1

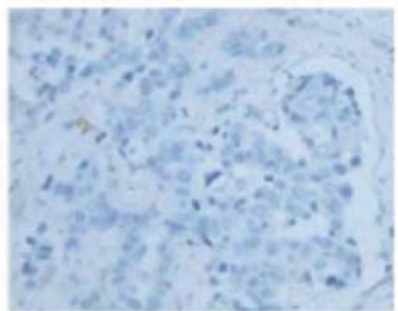

$\lg G$ control

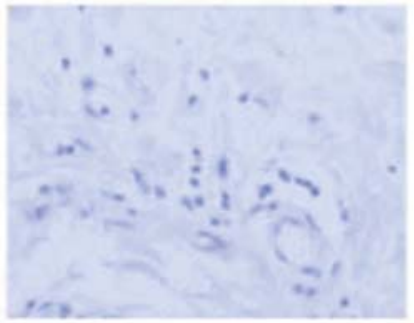

AlB-1

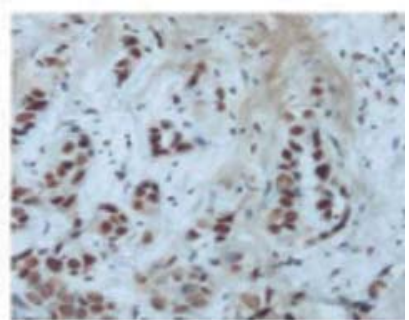

$\mathrm{SRC}-1$

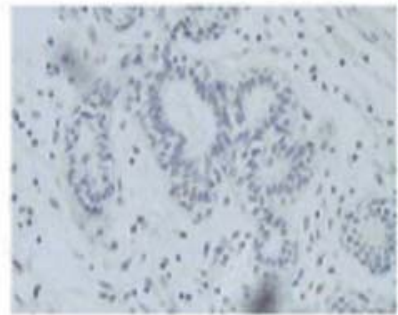

$\lg G$ control

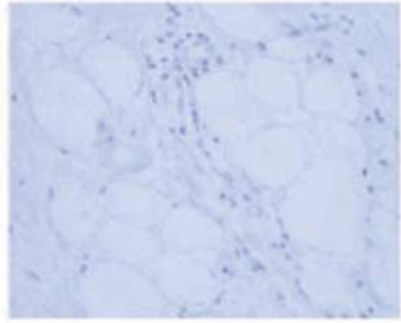

SRC-1

Figure 1 PEA3, AIB1, and SRC-1 protein expression in paraffin wax embedded invasive breast carcinoma and normal breast tissue specimens. (A) Immunohistochemical localisation of the HER2 transcription factor PEA3 and the 160 coactivators AIB1 and SRC-1 in primary breast cancer counterstained with haematoxylin and matched lgG controls (original magnification, $\times 200$ ). (B) PEA3, AIB1, and SRC-1 protein expression was absent in normal breast tissue (original magnification, $\times 200$ ).
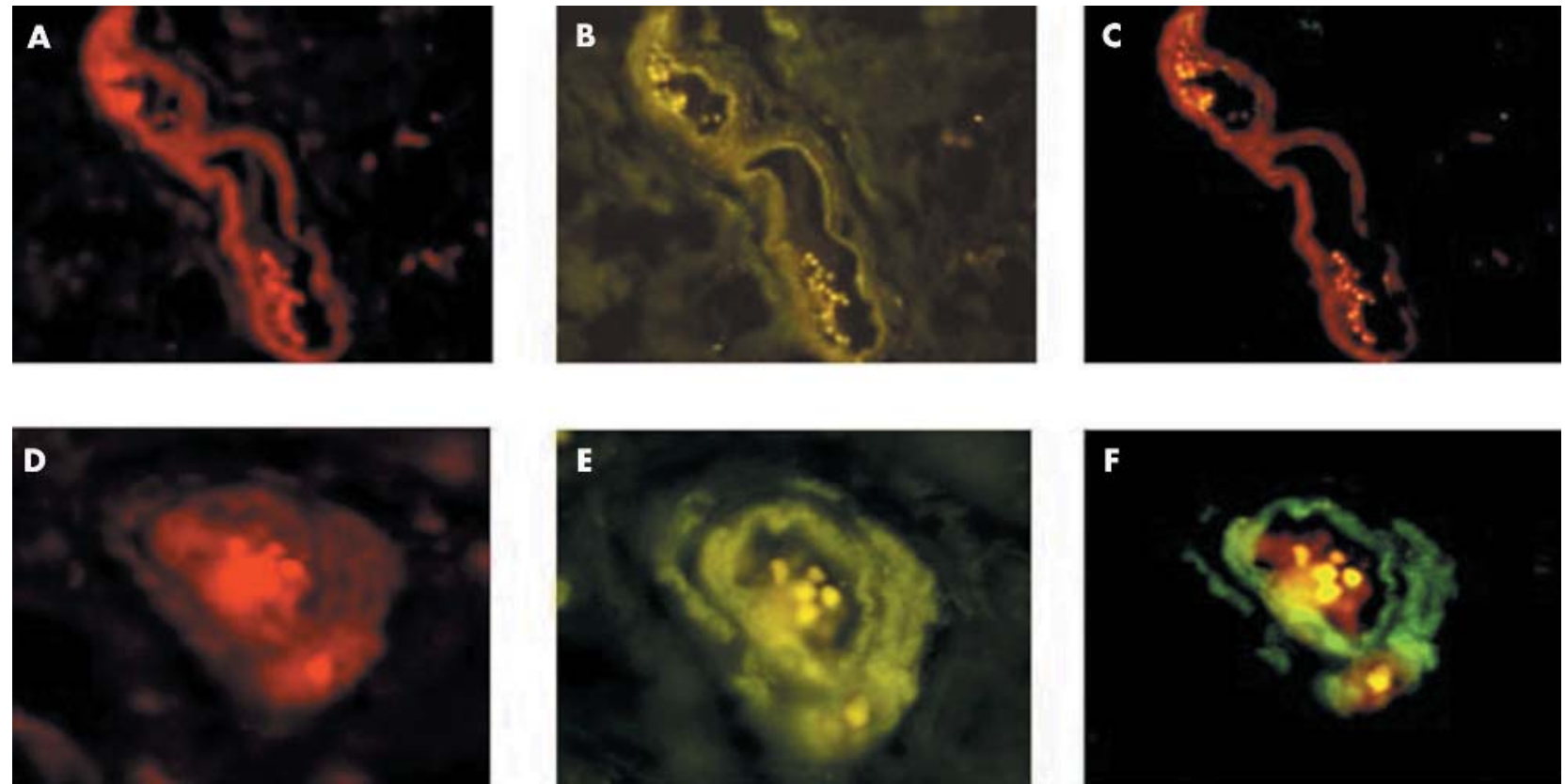

Figure 2 Colocalisation of PEA3 with the coactivators AIB 1 and SRC-1 in invasive breast carcinoma. Immunofluorescent colocalisation of PEA3 with AIB1 (original magnification, $\times 100$ ) and PEA3 with SRC-1 (original magnification, $\times 200$ ). 
estimates of survival functions were used to illustrate significant predictors of survival time. A logistic regression model was fitted to analyse the presence or absence of disease recurrence. SAS statistical software, Version 6, Fourth Edition (SAS Institute Inc 1989) was used to perform these analyses.

\section{RESULTS}

\section{Patient characteristics}

Table 1 shows the patient characteristics for the entire population and for HER2 positive and HER2 negative groups. The mean (SD) probability of survival beyond five years for the overall group was found to be $0.6146(0.0825)$. The two groups were matched for age and tumour size. There was no difference in terms of axillary lymph node status between the two groups; however, patients who expressed HER2 were found to have higher grade tumours (grade III $v$ non-grade III; $\mathrm{p}=0.0162$; table 1 ).

\section{PEA3, SRC-1, and AIB 1 expression in breast tumours} of known HER2 status

The transcription factor PEA3 and the pl60 coactivator proteins, AIBl and SRC-1, were localised within paraffin wax embedded human breast tissue using immunohistochemistry. Strong positive staining for PEA3 was detected within the nuclei of invasive ductal and invasive lobular breast tumour epithelial cells, whereas expression within the cytosol was negligible (fig 1A). Both AIBI and SRC-1 were expressed in the nuclei of breast epithelial cells, predominantly in those of the duct (fig lA). Expression of AIBl was more widespread within the tumour compared with that of SRC-1; this was reflected in the Allred scoring for the coactivators, where the median score over two observers for AIBl was 5 (0-8), compared with a median score of $1.5(0-4)$ for SRC-1 $(p<0.0001)$. Normal breast tissue did not express PEA3, AIB1, or SRC-1 (fig 1B). Immunofluorescence was undertaken to determine whether the HER2 transcription factor PEA3 could be localised to the same breast tumour cell as the steroid coregulators AIBI and SRC-1. PEA3 colocalised with both AIBl and SRC-1 within a subset of breast tumour epithelial cells (fig 2). Where PEA3 and AIB1 or SRC-1 were expressed within the same breast tissue, cellular coexpression of the transcription factor and the coregulators was seen in more than $70 \%$ of the positive tumour epithelial cells.

There was no relation between ER $\alpha$ and HER2 status, although ER $\beta$ was found to associate inversely with HER2. The rate of expression of PEA3 was significantly higher in HER2 positive patients than in those who did not express the oncogene (69\% $v 34 \% ; p=0.0486)$. AIBl and SRC-1 were both more frequently expressed in HER2 positive than in HER2 negative patients ( $p=0.0444$ and $p=0.0012$, respectively; table 2 ).

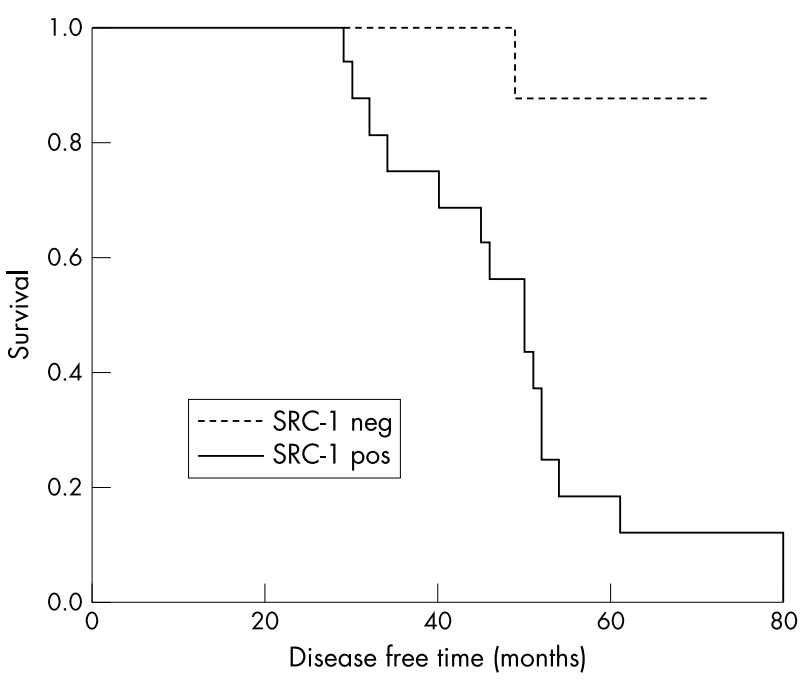

Figure 3 Kaplan-Meier estimates of disease free survival time according to SRC-1 expression.

\section{PEA3, AIB 1, and SRC-1 correlations with clinical variables in HER2 positive patients}

In the HER2 positive patient population, PEA3 expression correlated with the coactivator protein SRC-1 $(\mathrm{p}=0.0354)$, but not AIBl $(p=0.4159)$. Both SRC-1 and PEA3 were associated with recurrence on univariate analysis $(\mathrm{p}<0.0001$ and $\mathrm{p}=0.0354$, respectively $)$. On multivariate analysis, using a Cox proportional hazards model, SRC-1 was significantly associated with disease free survival time $(p=0.0066)$, as reflected in the Kaplan-Meier estimates of survival (fig 3). Moreover, the risk of recurrence for HER2 positive tumours with raised SRC-1 expression was 16.82, compared with SRC-1 negative tumours, when grade and ER status are considered equal. Using a logistic regression model, SRC-1 was also found to be a significant predictor of the time to disease recurrence $(p<0.0001)$. However, of interest, in the cohort of HER2 positive patients, there was no association detected between ER $\alpha$ expression and SRC-1 and an inverse association was seen between ER $\beta$ and SRC-1.

\section{DISCUSSION}

The transcriptional activity of ER is dependent both on its recruitment and interaction with coregulatory proteins. Selective ER modulators, such as tamoxifen, inhibit cancer growth through the competitive antagonism of the ER. Altered concentrations and perhaps activity of coactivator proteins at the ER-oestrogen response element complex may be important in the alteration of the agonist/antagonist profile of ER modulators in resistant tumours. In the breast, the pl60 family of coactivators, including AIBl and SRC-1,

Table 2 Recurrence rates and protein expression of ER $\alpha$, ER $\beta$, PEA3, AIB1, and SRC-1 in HER2 positive versus HER2 negative patients

\begin{tabular}{lllll}
\hline & Total group (70) & HER2 positive (\%) & HER2 negative (\%) & p Value (Bonferroni's adjusted p value) \\
\hline Recurrence & 19 & $16(46 \%)$ & $3(9 \%)$ & $0.0009(0.0054)$ \\
ER $\alpha$ positive & 60 & $28(80 \%)$ & $32(91 \%)$ & $0.3059(1.0000)$ \\
ERß positive & 37 & $11(31 \%)$ & $26(74 \%)$ & $0.0007(0.0042)$ \\
PEA3 positive & 36 & $24(69 \%)$ & $12(34 \%)$ & $0.0081(0.0486)$ \\
AIB1 positive & 40 & $26(74 \%)$ & $14(40 \%)$ & $0.0074(0.0444)$ \\
SRC-1 positive & 18 & $16(46 \%)$ & $2(6 \%)$ & $0.0002(0.0012)$ \\
\hline
\end{tabular}

Statistical analysis was performed using the Fisher's exact test. The $p$ values were adjusted for multiple comparisons using Bonferroni's inequality. ER, oestrogen receptor. 
has been implicated in ER signalling and tumour progression. ${ }^{15}{ }^{16}$ Expression of AIBl has been previously demonstrated exclusively in tumour versus normal breast tissue, whereas expression of SRC-1 has been shown in a subset of normal breast tissues adjacent to the tumour. ${ }^{17}$ In our study, no expression of either of these coactivator proteins was seen in normal reduction mammoplasties. There is little consensus within the literature, however, with regard to correlations between the expression of these coactivator proteins and resistance to endocrine treatment. ${ }^{16} 1819$ The specificity and activity of the coactivator proteins is thought to be regulated by intracellular signalling cascades, including phosphorylation via the MAP kinase pathway. ${ }^{10}$ In our study, we have demonstrated significant correlations between coactivator proteins and resistance to endocrine treatment in patients who overexpress the tyrosine kinase receptor HER2. However, based on our restrictive patient selection criteria, definitive clinical implications should be interpreted with caution.

Overexpression of the EGFR family member HER2 has been associated both experimentally and in clinical studies with tamoxifen resistance. Transcriptional regulation of this growth factor receptor is thought to be controlled, at least in part, by the Ets family of transcription factors, including PEA3. In the breast, PEA3 expression has previously been observed exclusively in tumour tissue. ${ }^{20}$ Consensus binding sites for PEA3 have been identified on the promoter region of the HER2 gene. ${ }^{21}$ Although the precise role of PEA3 in the transcription of HER2 remains to be fully elucidated, ${ }^{45}$ molecular and clinical studies have suggested a positive role for PEA3 in HER2 mediated breast tumorigenesis. ${ }^{67}$ Consistent with this, we found that the expression of the PEA3 transcription factor was associated with that of HER2 in our cohort of patients with breast tumours. Furthermore, in our cohort of HER2 positive patients with breast cancer, PEA3 was significantly associated with disease recurrence in patients on endocrine treatment.

\section{"We have described a positive correlation between HER2 and its transcriptional mediator PEA3 and the coactivator protein SRC-1, implicating SRC-1 in HER2 mediated resistance to endocrine treatment"}

It has been suggested that HER2 plays an essential role in endocrine sensitivity by activating the MAP kinase cascade, resulting in the phosphorylation, not only of ER, but also of coregulatory proteins. Previous studies by Bouras et al have described a significant correlation between AIBI mRNA and HER2 status. ${ }^{22}$ Here, the expression of the pl60 coactivators AIBl and SRC-1 was associated with HER2 expression; moreover, expression of the HER2 transcription factor PEA3 was also associated with SRC-1 in the HER2 positive population. Immunofluorescence studies revealed PEA3 coexpression with AIBl and SRC-1. Although this observation does not provide direct evidence of linkage between these factors, it does provide support for an association

\section{Take home messages}

- Patients with breast cancer who have high expression of both HER2 and SRC-1 have a greater probability of recurrence on endocrine treatment compared with those who are HER2 positive but SRC-1 negative

- SRC-1 may be an important predictive indicator and therapeutic target in breast cancer between PEA3 and pl60 proteins. A recent study by Osborne et al found that tumours with high expression of both AIBl and HER2 have a poor response to tamoxifen. ${ }^{10}$ In our study, we failed to find a correlation between AIBl and recurrence on endocrine treatment in HER2 positive patients. This discrepancy may be caused, at least in part, by our restricted patient population and the nature of our patient selectionpatients with HER2 positive tumours were matched with HER2 negative patients, which resulted in a relatively high recurrence rate. We found that the SRC-1 protein was significantly associated with insensitivity to endocrine treatment; this observation contrasts with that previously reported by Berns et al, which described an association between SRC-1 and a favourable response to tamoxifen. ${ }^{23}$ However, that study examined SRC-1 mRNA values in a limited number of tumour samples. In our cohort of patients with breast cancer, we found that the probability of tumour recurrence on endocrine treatment was significantly higher in patients who expressed HER2 in combination with SRC-1 than in those who expressed HER2 alone. These clinical ex vivo observations support previous molecular in vitro data linking SRC-1 and HER2 in human breast cancer. ${ }^{24}$ We have previously reported that SRC-1 can interact with both isoforms of ER to modulate transcription. ${ }^{12}$ However, in our present study, an inverse relation was seen between ER $\beta$ and SRC-1 expression, and there was no association detected between ER $\alpha$ expression and SRC-1 in our HER2 patient population. These observations raise the possibility that the pl60 proteins may interact with transcription factors other than ER to modulate gene transcription. Of interest, recent reports have described an interaction between the pl60 protein and the MAP kinase activated Ets transcription factor family..$^{25}$ These interactions may be relevant to the development of endocrine resistance, although in depth clinical and molecular studies are required to elucidate this complex transcription factor-coactivator association.

The data reported here have described a positive correlation between HER 2 and its transcriptional mediator PEA3 and the coactivator protein SRC-1, implicating SRC-1 in HER2 mediated resistance to endocrine treatment. These results suggest that coactivator proteins, including SRC-1, may serve as essential mediator proteins, bridging the crosstalk between the growth factor/kinase and steroid signalling pathways. These coregulatory proteins may serve not only as a complement to HER2 as a predictor of response to endocrine treatment, but may also prove attractive therapeutic targets, lying as they do at the crossroads of the kinase and steroid pathways.

\section{Authors' affiliations \\ F J Fleming, E Myers, E W McDermott, N J O'Higgins, A D K Hill,} L S Young, Department of Surgery, Saint Vincent's University Hospital, Elm Park, Dublin 4, Ireland

G Kelly, The Department of Statistics, University College Dublin, Dublin 4, Ireland

T B Crotty, A D K Hill, Department of Pathology, Saint Vincent's University Hospital

F J Fleming, E Myers, A D K Hill, L S Young, The Conway Institute, University College Dublin

The first two authors contributed equally to this work

\section{REFERENCES}

1 Slamon DJ, Clarke GM, Wong SG, et al. Studies of the HER2/neu protooncogene in human breast and ovarian cancer. Science 1987;244:707-12.

2 Muss HB, Thor AD, Berry DA, et al. C-erbB2 expression and response to adjuvant therapy in women with node-positive early breast cancer. N Engl J Med 1994;330:1260-6.

3 Wang SC, Zhang L, Hortobagyi GN, et al. Targeting HER2: recent developments and future directions in breast cancer patients. Semin Oncol 2001;28:21-9. 
4 Scott GK, Chang CH, Erny KM, et al. Ets regulation of the erbB2 promoter. Oncogene 2000;18:6490-502.

5 Xing X, Wang SC, Xia W, et al. The Ets protein PEA3 suppresses HER2/neu overexpression and inhibits tumorigenesis. Nat Med 2000;6:189-95.

6 Benz CC, O'Hagen RC, Richter B, et al. HER2/Neu and ets transcription activator PEA3 are co-ordinately up-regulated in human breast cancer. Oncogene 1997;15:1513-23.

7 Shepherd T, Kockeritz L, Szrajber MR, et al. The PEA3 subfamily of ets genes are required for HER2/Neu mediated mammary oncogenesis. Curr Biol 2001;11:1739-48.

8 Benz CC, Scott GK, Saurp JC, et al. Estrogen dependant, tamoxifen resistant tumorigenic growth of MCF-7 cells transfected with HER2/neu. Breast Cancer Res Treat 1993;24:85-95.

9 Wright C, Nicholson S, Angus B, et al. Relationship between c-erbB2 protein product expression and response to endocrine therapy in advanced breast cancer. Br J Cancer 1992;65:118-21.

10 Osborne CK, Bardou V, Hopp TA, et al. Role of the estrogen receptor coactivator AIB1 (SRC-3) and HER2/neu in tamoxifen resistance in breast cancer. J Natl Cancer Inst 2003;95:353-61.

11 Anzick SL, Kononen J, Walker RL, et al. AIB1, a steroid receptor co-activator amplified in breast and ovarian cancer. Science 1997;277:965-8.

12 Fleming FJ, Hill ADK, McDermott EW, et al. Differential recruitment of coregulator proteins SRC-1 and SMRT to the estrogen receptor-estrogen response element by beta-estradiol and 4 -hydroxytamoxifen in human breast cancer. J Clin Endocrinol Metab 2004;89:375-83.

13 Harvey JM, Clarke GM, Osborne CK, et al. Estrogen receptor status by immunohistochemistry is superior to the ligand binding assay for predicting response to adjuvant endocrine therapy in breast cancer. J Clin Oncol 1999:17:1474-82.

14 Fleiss JL. Introduction. In: The design and analysis of clinical experiments. New York: John Wiley and Sons, 1986.

15 Kraichely DM, Sun J, Katzenellenbogen JA, et al. Conformational changes and co-activator recruitment by novel ligands for estrogen receptor-alpha and estrogen receptor-beta: correlations with biological character and distinct differences among SRC co-activator family members. Endocrinology 2000; 14:3534-45.

16 Takimoto GS, Graham JD, Jackson TA, et al. Tamoxifen resistant breast cancer: coregulators determine the direction of transcription by antagonistoccupied steroid receptors. J Steroid Biochem Mol Biol 1999;69:45-50.

17 Hudelist G, Czerwenka K, Kubista E, et al. Expression of sex steroid receptors and their co-factors in normal and malignant breast tissue: $A I B 1$ is a carcinoma specific co-activator. Breast Cancer Res Treat 2003;78:193-204.

18 Murphy LC, Simon SLR, Parkes A, et al. Altered expression of estrogen receptor coregulators during breast tumorigenesis. Cancer Res 2000:60:6266-71.

19 Murphy LC, Leygue E, Niu Y, et al. Relationship of coregulator and estrogen receptor isoform expression to de novo tamoxifen resistance in human breast cancer. Br J Cancer 2002;87:1411-16.

20 Kinoshita J, Kitamura K, Tanaka S, et al. Clinical significance of PEA3 in human breast cancer. Surgery 2002;131:222-5.

21 White MR, Hung MC. Cloning and characterisation of the mouse neu promoter. Oncogene 1992;7:677-83.

22 Bouras T, Southey MC, Venter DJ. Over expression of the steroid receptor coactivator AIB1 in breast cancer correlates with the absence of estrogen and progesterone receptors and positivity for p53 and HER2neu. Cancer Res 2001;61:903-7.

23 Berns EM, Van Staveren IL, Klijn JG, et al. Predictive value of SRC-1 for tamoxifen response of recurrent breast cancer. Breast Cancer Res Treat 1998;48:87-92.

24 Newman SP, Bates NP, Vernimmen D, et al. Cofactor competition between the ligand bound oestrogen receptor and an intron 1 enhancer leads to oestrogen repression of ERBB2 expression in breast cancer. Oncogene 2000;19:490-7.

25 Goel A, Janknecht R. Concerted activation of ETS protein ER81 by p160 coactivators, the acetyltransferase $\mathrm{p} 300$ and the receptor tyrosine kinase HER2/Neu. J Biol Chem 2004;270:14904-16. 